A\&L

ISSN 2709-0205

Vol 2 Issue 2 (2021)

\title{
Impact of COVID-19 on the signs of human ageing
}

\author{
Anatoly V. Pisaruk, Natali M. Koshel, Ludmila V. Pysaruk, Valeri B. Shatilo \\ D.F. Chebotarev Institute of Gerontology NAMS of Ukraine, Kiev, Ukraine
}

https://dx.doi/10.47855/jal9020-2021-2-3

Correspondence: avpisaruk54@gmail.com

Received: 10.05.2021; Accepted: 09.07.2021; Published: 12.07.2021

\begin{abstract}
The symptoms of post-COVID-19 syndrome (PPCS) are nonspecific and similar to those signs of aging. Therefore, it was suggested that those who have recovered from COVID-19 have accelerating the aging of the body. The aim of this work was to assess the influence of COVID-19 on the frequency of signs of aging. To collect the data, there was used a developed questionnaire, which included 17 signs of aging. There were received the answers from 306 people at the age from 30 to 70 years old. Among them, 98 people have had COVID-19. The average age of the people in the post-COVID-19 and COVID-19-free groups was 51,3 $\pm 1,65$ and 50,4 $\pm 1,17$, respectively ( $p>0,05$ ). The frequency of 14 signs of aging was significantly $(\mathrm{p}<0,01)$ higher among post-COVID-19 people. Particularly significant differences between post-COVID-19 and COVID-19-free were in the group of people under the age of 50 years old. The data, received by us, allowed to develop a computational procedure for diagnosing of PPCS. The task of attributing of the surveyed people to the post-COVID-19 and COVID-19-free group in accordance with the presence of certain symptoms was solved with the help of the Bayesian method in Gubler's modification, multiple logistic regression and neural network analysis. The developed diagnostic procedures can be used for the identification of the people with a high probability of the developing of PPCS.
\end{abstract}

Keywords: rate of aging, post-COVID-19 syndrome, questionnaire

The problem of the relationship between COVID-19 and aging is insufficiently explored. The mortality from COVID-19 grows exponentially with the age [1,2]. This relationship is similar to the relationship between cardiovascular mortality and age. Age-related diseases are the predictors of mortality from COVID-19. The mortality from COVID-19 is higher among the people with age-related diseases such as coronary artery disease, arterial hypertension, diabetes mellitus of the second type and obesity, apparently because these diseases are associated with the accelerated aging [3-5]. It is known that the death rate from COVID-19 among the men is twice as high as among the women [6]. This may be due to the fact that men are getting old faster than women and their biological age (BA) is higher at any chronological age. COVID-19 somewhere about doubles the aging-related risk of death at a given age. Vulnerability to COVID-19 is related to the rate of aging, depends on BA and age-related diseases. BA has been shown to be a better predictor of mortality from COVID-19 than chronological age (CA) [7, 8]. Obesity is an important vulnerability factor to COVID-19 [9]. Obesity is associated with many age-related diseases and is a manifestation of accelerated metabolic aging. Post-COVID Syndrome (PPCS) is characterized by a number of features that take place during aging. There is a decrease in the functions of the cardiovascular, nervous systems, lungs, endothelial dysfunction, an increase in the tendency to thrombus formation [10]. It can be suggested that COVID-19 accelerates the aging of the body. Thus, in a recently published study [11], BA of 117 people who had recovered from COVID-19 (post-COVID-19) and 144 volunteers (COVID-19-free) were determined using the most accurate modern method for 
measuring BA - methylation of certain DNA sites (DNAmAge) [12]. For the calculation of BA, the authors used the Bekaert's algorithm [13]. The results of this study indicate a significant increase in BA among people after COVID-19 (for $10.45 \pm 7.29$ years, $\mathrm{p}<0.0001$ ). A significant reduction in telomeres was also found among the post-COVID-19 cohort of people $(p<0.0001)$. The revealed changes were more pronounced among the people under the age of 60 years old. It is not known at this time the extent to which BA changes are reversible among the people with PPCS.

Previously we have developed a questionnaire for a simple and quick assessment of the biological age of people [14]. This questionnaire includes a set of signs of aging. It turned out that many of these features coincide with the features characterizing PPCS.

The aim of the work was to assess the impact of COVID-19 on the frequency of signs of aging with the help of the questionnaire.

\section{Materials and methods}

An on-line method developed by Google (the so-called Google forms) was used to collect the data. This method allows you to distribute the questionnaire on the Internet and get information about the answers to its questions in the form of a google table on Google Drive. We have developed a Google form of the questionnaire that includes 17 signs of aging. This questionnaire can be downloaded to your browser at the following link https://forms.gle/Jjn4e385PcdwPU1G7. Links to the questionnaire were posted in the gerontological Facebook groups and distributed via Viber among the staff of the Institute of Gerontology of the National Academy of Medical Sciences of Ukraine and their acquaintances. The respondents were asked to choose the answer "Yes" or "No" for each item of the questionnaire. The examined people were informed that the questions were about health changes during the last year.

The answers were received from 306 people at the age from 30 to 70 years old. Among them, 98 people have had COVID-19. The diagnosis was done on the basis of positive tests for the SARS-CoV-2 antigen. $62 \%$ of the surveyed people transferred the disease in a mild form and $38 \%$ in the form of moderate severity. $45 \%$ of people in the post-COVID-19 group had been ill in the period from 1 to 3 months before the survey, $55 \%$ - from 3 to 6 months before the survey. The average age in the groups of people with post-COVID-19 and COVID-19-free did not differ significantly and was $51.3 \pm 1.65$ and $50.4 \pm$ 1.17 , respectively. The frequency of occurrence of signs of aging in post-COVID-19 and COVID-19-free groups of people and their diagnostic coefficients (DC) were calculated. DC of the sign of aging was calculated as the decimal logarithm $(\log 10)$ of the ratio of the sign frequencies in these two groups of people, multiplied by 10. Such an assessment of the informativeness is based on the Bayesian probability theory formula modified by E.V. Gubler [15]. The solution of the diagnostic problem by this method consists of summing of the DC of those symptoms that occur at a given person and comparing the resulting sum with the threshold one.

Statistical processing of the obtained data was carried out with the help of Excel 2007 and Statistica 7 (StatSoft, USA) programs. Standard statistical procedures, including variation and regression analyses, were used.

\section{Results and discussion}

Table 1 shows the frequencies of signs of aging in two groups of people: post-COVID-19 and COVID-19-free. The significance of the differences of frequency in the groups was assessed using the Chisquare criterion. In 14 out of 17 signs of aging, the differences between the groups are significant. Among the people who have had COVID-19, these signs of aging are much (some of them in several times) more common. Hereof, it can be assumed that COVID-19 accelerates age-related changes in the body.

We also compared the frequency of signs of aging among the people with post-COVID-19 and COVID-19-free in the age groups before and after 50 years (Tabl. 2, 3). For the people under the age of 50 years old, the differences are significant in 13 out of 17 signs, and for the people over 50 years old, only in 5 signs of aging. These data give an evidence about more rapid development of age-related changes after being ill for COVID-19 among young people. 
Table 1

Frequency of indicators of aging (\%) in groups with post-COVID-19 and COVID-19-free and their diagnostic coefficients (DC)

\begin{tabular}{|l|l|l|l|l|}
\hline Indicators of aging & $\begin{array}{l}\text { post- } \\
\text { COVID-19 }\end{array}$ & $\begin{array}{l}\text { COVID-19- } \\
\text { free }\end{array}$ & P & DC \\
\hline Appeared an increased fatigue & 85.9 & 53.1 & 0.0001 & 2.09 \\
\hline You find it difficult to put on socks, shoes & 54.9 & 23.8 & $<0.0001$ & 3.62 \\
\hline It became necessary afternoon sleep & 47.9 & 20.0 & $<0.0001$ & 3.79 \\
\hline You started to sleep badly at night & 63.4 & 41.5 & 0.0012 & 1.84 \\
\hline You had begun to fall asleep in front of the TV & 40.8 & 26.9 & 0.0619 & 1.81 \\
\hline Appeared shortness of breath when walking fast & 57.7 & 29.2 & $<0.0001$ & 2,96 \\
\hline You have started to walk slower & 45.1 & 21.5 & 0.0001 & 3.21 \\
\hline You have begun to forget names, words & 73.2 & 392 & $<0.0001$ & 2.71 \\
\hline Your hearing had worsened & 22.5 & 15.4 & 0.082 & 1.66 \\
\hline Your vision had worsened significantly & 50.7 & 39.2 & 0.1403 & 1.11 \\
\hline A wobbly gait had appeared & 29.6 & 10.0 & 0.0032 & 4.71 \\
\hline Your weight has increased by more than 2 kg & 56.3 & 39.2 & 0.004 & 1.57 \\
\hline $\begin{array}{l}\text { Your blood pressure has increased by more than } 10 \\
\text { mm Hg }\end{array}$ & 47.9 & 16.2 & $<0.0001$ & 4.72 \\
\hline There was anxiety, depressed mood & 63.4 & 36.9 & 0.0001 & 2.35 \\
\hline $\begin{array}{l}\text { Slow performance or unwillingness to perform } \\
\text { habitual mental or physical work }\end{array}$ & 73.2 & 50.8 & 0.0002 & 1.59 \\
\hline There were palpitations, interruptions & 47.9 & 22.3 & 0.0001 & 3.32 \\
\hline Discomfort in the joints, back, stiffness & 63.4 & 46.2 & 0.0131 & 1.38 \\
\hline
\end{tabular}

Table 2

Frequency of indicators of aging (\%) in groups with post-COVID-19 and COVID-19-free (age $<50$ years)

\begin{tabular}{|l|l|l|l|}
\hline Indicators of aging & $\begin{array}{l}\text { post- } \\
\text { COVID-19 }\end{array}$ & $\begin{array}{l}\text { COVID-19- } \\
\text { free }\end{array}$ & P \\
\hline Appeared an increased fatigue & 88.6 & 46.2 & $\mathbf{0 . 0 0 0 1}$ \\
\hline You find it difficult to put on socks, shoes & 48.6 & 18.5 & $\mathbf{0 . 0 0 3 3}$ \\
\hline It became necessary afternoon sleep & 54.3 & 16.9 & $\mathbf{0 . 0 0 0 3}$ \\
\hline You started to sleep badly at night & 60.0 & 26.2 & $\mathbf{0 . 0 0 1 9}$ \\
\hline You had begun to fall asleep in front of the TV & 28.6 & 21.5 & 0.5892 \\
\hline Appeared shortness of breath when walking fast & 57.1 & 24.6 & $\mathbf{0 . 0 0 2 6}$ \\
\hline You have started to walk slower & 40.0 & 15.4 & $\mathbf{0 . 0 1 2 3}$ \\
\hline You have begun to forget names, words & 68.6 & 43.1 & $\mathbf{0 . 0 2 6 1}$ \\
\hline Your hearing had worsened & 14.3 & 10.8 & 0.8465 \\
\hline Your vision had worsened significantly & 54.3 & 26.2 & $\mathbf{0 . 0 1 0 0}$ \\
\hline A wobbly gait had appeared & 28.6 & 7.7 & $\mathbf{0 . 0 1 2 6}$ \\
\hline Your weight has increased by more than 2 kg & 54.3 & 36.9 & 0.1440 \\
\hline Your blood pressure has increased by more than 10 mm Hg & 51.4 & 4.6 & $\mathbf{0 . 0 0 0 0}$ \\
\hline There was anxiety, depressed mood & 74.3 & 35.4 & $\mathbf{0 . 0 0 0 5}$ \\
\hline $\begin{array}{l}\text { Slow performance or unwillingness to perform habitual mental } \\
\text { or physical work }\end{array}$ & 74.3 & 47.7 & $\mathbf{0 . 0 1 8 8}$ \\
\hline There were palpitations, interruptions & 51.4 & 15.4 & $\mathbf{0 . 0 0 0 3}$ \\
\hline Discomfort in the joints, back, stiffness & 62.9 & 41.5 & 0.0681 \\
\hline
\end{tabular}


Table 3

Frequency of indicators of aging (\%) in groups with post-COVID-19 and COVID-19-free (age $\geq 50$ years)

\begin{tabular}{|l|l|l|l|}
\hline Indicators & $\begin{array}{l}\text { post- } \\
\text { COVID-19 }\end{array}$ & $\begin{array}{l}\text { COVID-19- } \\
\text { free }\end{array}$ & P \\
\hline Appeared an increased fatigue & 86.5 & 60.9 & $\mathbf{0 . 0 1 3 1}$ \\
\hline You find it difficult to put on socks, shoes & 59.5 & 29.2 & $\mathbf{0 . 0 0 5 4}$ \\
\hline It became necessary afternoon sleep & 40.5 & 23.1 & 0.1020 \\
\hline You started to sleep badly at night & 64.9 & 56.9 & 0.5643 \\
\hline You had begun to fall asleep in front of the TV & 51.4 & 32.3 & 0.0924 \\
\hline Appeared shortness of breath when walking fast & 62.2 & 33.8 & $\mathbf{0 . 0 1 0 4}$ \\
\hline You have started to walk slower & 48.6 & 27.7 & 0.0556 \\
\hline You have begun to forget names, words & 75.7 & 35.4 & $\mathbf{0 . 0 0 0 2}$ \\
\hline Your hearing had worsened & 29.7 & 20.0 & 0.3837 \\
\hline Your vision had worsened significantly & 45.9 & 52.3 & 0.6804 \\
\hline A wobbly gait had appeared & 35.1 & 12.3 & $\mathbf{0 . 0 1 2 9}$ \\
\hline Your weight has increased by more than 2 kg & 56.8 & 41.5 & 0.2026 \\
\hline Your blood pressure has increased by more than 10 mm Hg & 43.2 & 27.7 & 0.1665 \\
\hline There was anxiety, depressed mood & 51.4 & 38.5 & 0.2910 \\
\hline $\begin{array}{l}\text { Slow performance or unwillingness to perform habitual } \\
\text { mental or physical work }\end{array}$ & 72.2 & 53.8 & 0.1105 \\
\hline There were palpitations, interruptions & 44.4 & 29.2 & 0.1867 \\
\hline Discomfort in the joints, back, stiffness & 62.2 & 50.8 & 0.3656 \\
\hline
\end{tabular}

The obtained data made it possible to develop a calculating procedure for recognizing of the condition of people who have recovered from COVID-19. The task was to classify all the interviewed people into post-COVID-19 and COVID-19-free by the presence of certain symptoms among them. To solve this problem, the Bayesian method modified by Gubler, multiple logistic regression and neural network analysis were used.

In accordance with the Bayes-Gubler algorithm, DC of aging signs were calculated (Tabl. 1). The diagnostic procedure consists of summing the DC values of all symptoms present in the patient. The resulting amount is compared with the threshold amount. If it exceeds the threshold, then it is concluded that the person may have had COVID-19. The threshold value of the sum is chosen in such a way so as to minimize the proportion of diagnostic errors. In accordance to our data, such a threshold is the sum DC= 16.8. At the same time, the accuracy of the correct recognition is $77.4 \%$, which is quite acceptable. For the same purpose, the method of logistic multiple regression was used with the exclusion of uninformative indicators.

$$
\mathrm{Y}=1.22 \times \mathrm{X} 1+1.93 \times \mathrm{X} 2+1.17 \times \mathrm{X} 3+0.94 \times \mathrm{X} 4+1.23 \times \mathrm{X} 5+1.32 \times \mathrm{X} 6-3.85
$$

X1 - you find it difficult to put on socks, shoes;

$\mathrm{X} 2$ - you have begun to forget names, words;

X3 - you have started to walk slower;

X4 - you started to sleep badly at night,

X5 - your blood pressure has increased by more than $10 \mathrm{~mm} \mathrm{Hg}$,

X6 - it became necessary afternoon sleep.

The accuracy of the model was $81.4 \%$. Belonging to the post-COVID-19 group is determined by the model with an accuracy of $70.7 \%$. Area under the curve AUC $=0.88(0.82-0.93), x 2$-foursquare $=82.7, \mathrm{P}<$ 0.001 . This model has good predictive power, $\mathrm{AUC}=0.88, \mathrm{P}<0.001$, which will allow to estimate with 
high reliability the probability of developing of PPCS depending on the level of independent indicators, that are included in the model.

To recognize the people who have had COVID-19, we also used neural network analysis. The task was to classify all the interviewed people in accordance with their answers to the questionnaire into two groups: post-COVID-19 and COVID-19-free. The best results were shown by the neural network MLP 38-13-2. We used the training algorithm - BFGS, Error function - Entropy, hidden activation - Tanh, Output activation - Softmax. As a result of training of the neural network on the people survey dataset, the accuracy of recognizing people who have had COVID-19 was $95.8 \%$.

The results of the testing of algorithms for recognizing people who have had COVID-19 indicate for the significant and reliable differences between post-COVID-19 and COVID-19-free groups of people. The developed diagnostic procedures can be used for the assessment of the probability of the developing PPCS among the people who have recovered from COVID-19.

\section{Conclusions}

The obtained data allow us to draw the following conclusions.

1. In people who have recovered from COVID-19, the frequency of signs of aging is significantly higher than in healthy people of the same age. This suggests that COVID-19 accelerates agerelated changes in the body.

2. The impact of COVID-19 on the frequency of the signs of aging is higher among the people who are at the age under 50 years old.

3. The accuracy of recognizing of the state of people after COVID-19 with the help of various mathematical methods is quite high $(77-95 \%)$, which can be used to assess the probability of the developing of PPCS among the people who have had COVID-19.

Author Contributions: All authors participated equally in writing this commentary.

Conflicts of Interest: The authors declare no conflict of interest.

\section{Information about Authors:}

Anatoly V. Pisaruk - DSc (Medicine), Head of the Laboratory for Mathematical Modeling of Aging Processes; https://orcid.org/0000-0001-5522-0172

Nataly M. Koshel - PhD (Biology), Researcher of the Laboratory for Mathematical Modeling of Aging Processes; https://orcid.org/0000-0003-1429-2326

Ludmila V. Pysaruk - Researcher of the Laboratory for Mathematical Modeling of Aging Processes, https://orcid.org/0000-0002-9604-036X

Valeri B. Shatilo - DSc (Medicine), Prof., Leading Researcher of the Department of Clinical Physiology and Pathology of Internal Organs, Deputy Director for Research; https://orcid.org/0000-0001-6420-000X 


\section{References}

1. Leung, C. Risk factors for predicting mortality in elderly patients with COVID-19: a review of clinical data in China. Mech Ageing Dev 2020, 188:111255. doi.org/10.1016/j.mad.2020.111255

2. Promislow, D.E. A geroscience perspective on COVID-19 mortality. J Gerontol A Biol Sci Med Sci. 2020. doi.org/10.1093/gerona/glaa09

3. Hussain, A.; Bhowmik, B.; do Vale Moreira, N.C. COVID-19 and diabetes: knowledge in progress. Diabetes Res Clin Pract 2020, 162:108142. doi.org/10.1016/j.diabres.2020.108142

4. Mehra, M.R.; Desai, S.S.; Kuy, S.; Henry, T.D.; Patel, A.N. Cardiovascular disease, drug therapy, and mortality in covid-19. N Engl J Med 2020. doi.org/10.1056/NEJMoa2007621

5. Zhang, J.; Wu, J.; Sun, $\mathrm{X}$; et all. Association of hypertension with the severity and fatality of SARSCoV-2 infection: a meta analysis. Epidemiol Infect 2020, 148:e106. oi.org/10.1017/S095026882000117X

6. Jin, J.M.; Bai, P.; He, W. et all. Gender differences in patients with COVID-19: focus on severity and mortality. Front Public Health. 2020, 8:152. doi.org/10.3389/fpubh.2020.00152

7. Lauc, G.; Sinclair, D. Biomarkers of biological age as predictors of COVID-19 disease severity. Aging 2020, 12:6490-91. doi.org/10.18632/aging.103052

8. Blagosklonny, M. From causes of aging to death from COVID-19. Aging 2020, 12, 11.

9. Palaiodimos, L.; Kokkinidis, D.G.; Li, W.; Karamanis, D. et all. Severe obesity, increasing age and male sex are independently associated with worse in-hospital outcomes, and higher in-hospital mortality, in a cohort of patients with COVID-19 in the bronx, new york. Metabolism 2020, 108:154262. doi.org/10.1016/j.metabol.2020.154262

10. Oronsky, B.; Larson, C.; Hammond, T.C.; Oronsky, A. A review of persistent postCOVID syndrome (PPCS). Clinical Reviews in Allergy \& Immunology 2021. doi.org/10.1007/s12016-021-08848-3

11. Mongelli, A.; Barbi, V.; Zamperla, M. et all. Evidence for Biological Age Acceleration and Telomere Shortening in COVID-19 Survivors. Int. J. Mol. Sci. 2021, 22(11), 6151; doi.org/10.3390/ijms22116151

12. Horvath, S. DNA methylation age of human tissues and cell types. Genome Biol 2013, 14, R115. doi.org/10.1186/gb-2013-14-10-r115

13. Bekaert, B.; Kamalandua, A.; Zapico, S.C.; Van De Voorde, W.; Decorte, R. Improved age determination of blood and teeth samples using a selected set of DNA methylation markers. Epigenetics 2015, 10, 922-930. doi.org/10.1080/15592294.2015.1080413

14. Pisaruk, A.V.; Shatilo, V.B.; Antonyuk-Scheglova, I.A.; Naskalova, S.S. Express diagnostics of accelerated human aging using the questionnaire. Aging and longevity 2020, 2, 82-88. dx.doi.org/10.47855/jaI9020-2020-2-4

15. Gubler E.V. Computational methods of analysis and recognition of pathological processes. Leningrad. Medicine. 1978. 